Tohoku J. Exp. Med., 2011, 224, 229-234

\title{
Transplantation of Adipose Stromal Cells Promotes Neovascularization of Random Skin Flaps
}

\author{
Lingling Sheng, ${ }^{1, *}$ Mei Yang, ${ }^{1,2, *}$ Hua Li, ${ }^{1}$ Zijing Du, ${ }^{1}$ Yiai Yang ${ }^{1}$ and Qingfeng $\mathrm{Li}^{1}$ \\ ${ }^{1}$ Department of Plastic \& Reconstructive Surgery, Shanghai Ninth People's Hospital, Shanghai Jiao Tong \\ University, School of Medicine, Shanghai, P.R. China \\ ${ }^{2}$ Division of Plastic Surgery, University of Mississippi Medical Center, Jackson, MS, USA
}

\begin{abstract}
The delivery of bone marrow-derived mononulear cells (BM-MNCs) has been proved to be effective at promoting neovascularization of ischemic skin flaps. However, the limited source of BM-MNCs restricts their clinical application. Stromal vascular fraction (SVF) contains a group of heterogeneous cells in the adipose tissue, including adipose tissue-derived stem cells, and it has abundant reserve in human body. In this study, we evaluated the therapeutic potential of SVF to promote neovascularization of random skin flaps. Female Wistar rats were randomly devided into three groups with 8 in each group and received allogeneic SVF, BM-MNCs and phosphate-buffered saline (PBS), respectively, before surgery. Two days after cell administration, a $10 \times 3 \mathrm{~cm}$ random skin flap was elevated. Flap survival, blood flow perfusion and capillary density were examined 7 days after surgery, and the relevant mechanism was also explored. Results showed that SVF group and BM-MNCs group had higher survival percentage $(72.2 \pm 2.0 \%$ and $76.4 \pm 3.1 \%$, respectively) as compared with the control group $(56.8 \pm 4.6 \%, P<0.05)$. Blood flow perfusion and capillary density of flap tissues in SVF and BM-MNCs groups were both improved. The expression levels of vascular endothelial growth factor (VEGF) and basic fibroblast growth factor (bFGF) were increased in flap tissues of SVF and BM-MNCs groups detected by ELISA. These results indicate that SVF could promote vascularization and increase flap survival probably by secreting VEGF and bFGF. The effect of transplantation of SVF on therapeutic angiogenesis of skin flaps is equivalent to that of BM-MNCs.
\end{abstract}

Keywords: bone marrow derived nuclear cells; neovascularization; paracrine; skin flap; stromal vascular fraction Tohoku J. Exp. Med., 2011, 224 (3), 229-234. (C) 2011 Tohoku University Medical Press

Skin flap necrosis caused by lack of blood supply and severe ischemia, especially in the distal part of flaps, is a common problem in clinic. If neovascularization could be improved before operation, tissue necrosis would be reduced.

Neovascularization of skin flap is a complicated process, which requires proliferation of endothelial cells and cooperation of various growth factors (Yang et al. 2010). Application of growth factors such as vascular endothelial growth factor (VEGF) (Zhang et al. 2003), basic fibroblast growth factor (bFGF) (Haws et al. 2001) and transforming growth factor- $\beta$ (TGF- $\beta$ ) (Nall et al. 1996) has been demonstrated a promising therapeutic method to improve survival percentage of ischemic flaps, but it is restricted by short half-life period and non-long lasting effect of growth factors. What is more, administration of a single growth factor may not yield significant effects (Henry et al. 2003).

In recent years, cell therapy attracts much attention and has been found to be an effective method. Stromal vascular fraction (SVF) of the adipose tissue contains a group of heterogeneous cells consisting of adipose tissue-derived stem cells (ADSCs), endothelial progenitor cells (EPCs), hematopoietic progenitors, $\mathrm{T}$ cells and anti-inflammatory cells (Zimmerlin et al. 2010). The effect of SVF mainly depends on ADSCs within it. It has been proved that ADSCs can secrete various growth factors relating to angiogenesis as well as anti-apoptosis (Rehman et al. 2004) and differentiate into endothelial cells both in vivo (Bunnell et al. 2008) and in vitro (Lu et al. 2008). Large quantity of SVF will surely play same roles as homogeneous ADSCs and could be used as a better option for implantation.

A positive role of SVF has been verified in ischemic hindlimb study (Sumi et al. 2007). However, its therapeutic effect on flap ischemia has remained unclear. In this study, the effect of SVF transplantation on skin flap viability was examined and the potential therapeutic mechanism was also

\footnotetext{
Received May 10, 2011; revision accepted for publication June 13, 2011. doi: 10.1620/tjem.224.229

*Lingling Sheng and Mei Yang contributed equally to the research and should beviewed as co-first authors.

Correspondence: Qingfeng Li, M.D., Ph.D, Department of Plastic \& Reconstructive Surgery, Shanghai Ninth People’s Hospital,

Shanghai Jiao Tong University, School of Medicine, 639 Zhizaoju Road, Shanghai, 200011, P.R. China.

e-mail: liqfliqf@yahoo.com.cn
} 
explored.

\section{Materials and Methods}

Animals and surgical procedures

All animal procedures in this study were approved by Shanghai Jiao Tong University Medical Center, Institutional Animal Care and Use Committee. All animals were maintained in specific-pathogenfree environments. The surgical procedures were performed under aseptic conditions.

\section{Cell isolation and preparation}

Twenty Wistar rats (3-week-old; male) as cell donors were sacrificed with $3 \%$ sodium pentobarbital. The inguinal fat pads were harvested and digested at $37^{\circ} \mathrm{C}$ for 60 minutes with $0.2 \%$ collagenase II (Sigma-Aldrich, St. Louis, MO, USA). The cell suspension was filtrated through $40 \mu \mathrm{m}$ filter, then mature adipocytes were separated from cell pellet. The pellet was treated with erythrocyte lysis buffer containing $154 \mathrm{mM} \mathrm{NH}_{4} \mathrm{Cl}, 10 \mathrm{mM} \mathrm{KHCO}_{3}$ and $0.1 \mathrm{mM}$ ethylenediamineteraacetic acid and washed in phosphate buffer saline (PBS; GIBCO-BRL, Grand Island, N.Y.) twice to remove red cell fragment. The harvested pellet was SVF. BM-MNCs were harvested from femora and tibias as already described (Yang et al. 2010). $5 \times 10^{7}$ cells were suspended respectively in $1 \mathrm{ml}$ PBS for injection into each random pattern skin flap.

\section{Random skin flaps model and cell transplantation}

Twenty-four female Wistar rats of $200 \mathrm{~g}$ were randomly divided into three groups with 8 rats in each group. A $10 \times 3 \mathrm{~cm}$ random skin flap was devised on the back of each rat. Cells suspensions of SVF or BM-MNCs were injected into the interspace between the dermal tissue and subcutaneous membrana carnosa along the axis from the base to the distant end evenly. The control group received $1 \mathrm{ml}$ PBS. The flaps were raised two days after injection in a sterile environment. The flaps include skin, subcutaneous membrana carnosa. A 0.3-mmthick silicon sheet was placed under the flap to prevent angiogenesis from the recipient bed. The flaps were then sutured back in situ with 3-0 silk suture.

\section{Flap assessment}

Seven days after the operation, the surviving area of flaps was grossly determined on the basis of appearance, color, and texture. Then, the area of surviving and necrotic zones was quantified by Image Pro Plus Software (version 6.0, Media Cybernetics). Results were expressed as percentages of surviving area in relation to the total flap surface area.

\section{Vascularization detection}

The neovascularization level of skin flaps was detected by intensity of blood flow perfusion and capillary density 7 days after surgery. In brief, blood perfusion was assessed with a Full-Field Laser Perfusion Imager (FLPI, Moor Instruments Ltd, Axminster, UK). Tissue sections from the same position of each group (in the surviving district near the demarcation of survival and necrosis) were collected. The tissues were embedded in paraffin for hematoxylineosin (H\&E) staining to detect capillaries. Neovascularization was assessed by averaging the number of capillaries in subcutaneous tissue in 10 randomly chosen fields $(100 \times$ magnification; measurements were performed by two blinded reviewers).
Detection of viability of engrafted cells

SVF and BM-MNCs were labeled with 1,1'-Dioctadecyl3,3,3',3'-Tetramethy- lindocarbocyanine (DiI; Sigma-Aldrich) for tracking according to previously established methods (Lu et al. 2008). The rest of the steps were as above. 7 days after operation, tissue with a size of $0.5 \times 0.5 \mathrm{~cm}$ in injected area was harvested. After fixed in paraffin and dehydrated in $30 \%$ sucrose solution, the tissues were embedded in optimal cutting temperature compound (OCT; Wako, Osaka, Japan) and cut into $10 \mu \mathrm{m}$ slices. The slices were observed under a fluorescence microscope to observe DiI-positive cells.

\section{Expression of VEGF and bFGF in flap tissues}

For detection of growth factors, enzyme-linked immunosorbent assay (ELISA) was applied. $0.5 \mathrm{~g}$ tissue in the above area from each group was collected ( $n=6$ for each group). The tissues were homogenized in $500 \mu$ lissue protein extraction reagent (CWBIO, Beijing, China) and $5 \mu$ PMSF (Sigma-Aldrich). After centrifuged at 10,000 $\mathrm{rpm}$ for $10 \mathrm{~min}$, the supernatant was collected for the assay of VEGF and bFGF using ELISA kit according to the manufacturer's instructions (R\&D, Minneapolis, USA). OD values were examined on a Microplate reader (DENLEY DRAGON, Labsystems).

\section{Statistical analysis}

All values were expressed as mean \pm S.D. One-way analysis of variance with least significant difference (LSD) was used to determine the significance of differences in multiple comparisons. Values of $P<0.05$ were considered statistically significant. All statistical analyses were performed using SPSS version 16.0 (SPSS, Inc., Chicago, I11).

\section{Results}

\section{Observation of flap survival}

At 7 days after the operation, there was a clear dividing line between surviving and necrotic area. The percentage of surviving tissue in SVF group $(72.2 \pm 2.0 \%)$ and BM-MNCs group $(76.4 \pm 3.1 \%)$ was statistically higher than that in the control group $(56.8 \pm 4.6 \%)$. No statistical difference was found between the SVF group and the BM-MNCs group, $P>0.05$ (Fig. 1).

\section{Effects of SVF and BM-MNCs on neovascularization of skin flaps}

The blood flow of the flaps was monitored by FLPI. The ranges of blood flow perfusion of the flaps were all coincident with general surviving areas in the three groups. There was no significant change of blood perfusion in the proximal part of skin flaps. In the control rat treated with PBS, the blood flow in ischemic zone reduced obviously. (Fig. 2).

Improvement of angiogenesis in flaps was observed in both SVF group and BM-MNCs group demonstrated by higher capillary density $(7.0 \pm 1.0$ and $6.8 \pm 1.0$ respectively) compared with the control group $(3.8 \pm 1.3), P<$ 0.05 . There was no statistical difference between SVF group and BM-MNCs group, $P>0.05$ (Fig. 3). 

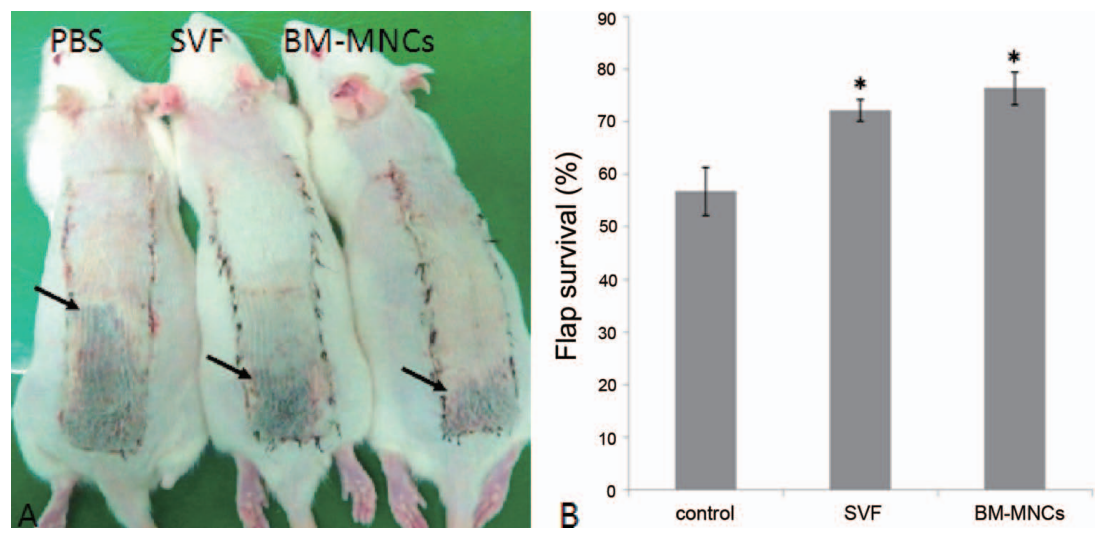

Fig. 1. Representative gross view of surviving area and statistical analysis.

(A) Representative gross view. Statistical analysis (B) indicates that the survival percentage of SVF group and BMMNCs group was higher than that of the control group 7 days postoperative, ${ }^{*} p<0.05$ (the arrows show the dividing line between necrotic and surviving area in the three groups).

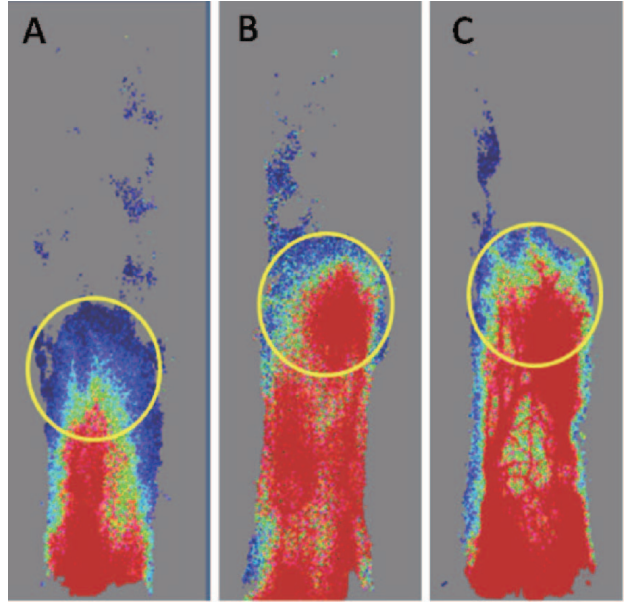

Fig. 2. Observation of blood flow perfusion.

Through laser perfusion image, difference of blood intensity among the three groups appeared in the ischemic area (in the yellow circles), which were near the necrotic area. In the images, red means higher blood flow perfusion than blue. Images $\mathrm{A}-\mathrm{C}$ showed blood perfusion images in control group, SVF group and BM-MNCs group respectively.

\section{Cell tracking}

At the seventh day, the slices were observed with a fluorescence microscope to observe the viability of engrafted cells. The DiI-labeling SVF and BM-MNCs released specific red fluorescence, and stayed mainly in subcutaneous tissue (Fig. 4).

\section{Protein levels of VEGF and bFGF in skin flaps}

The VEGF protein level was found much higher in SVF and BM-MNCs treated flap tissues $(178.66 \pm 4.92 \mathrm{pg} /$ $\mathrm{ml}$ and $189.14 \pm 4.67 \mathrm{pg} / \mathrm{ml}$ respectively) than that in the control group $(89.48 \pm 4.85 \mathrm{pg} / \mathrm{ml}), P<0.05$ (Fig. 5A). Similarly, bFGF in SVF group $(56.03 \pm 2.93 \mathrm{pg} / \mathrm{ml})$ and BM-MNCs group $(51.69 \pm 1.99 \mathrm{pg} / \mathrm{ml})$ were both higher than the control group $(32.51 \pm 1.31 \mathrm{pg} / \mathrm{ml}), P<0.05$ (Fig.
5B). However, the protein expressions of VEGF and bFGF both showed no differences between SVF group and BM-MNCs group.

\section{Discussion}

Skin flap repair is a routine treatment for tissue defects caused by wounds or tissue excisions. To improve the survival of transplanted skin flaps, surgical delay is always used as a common method to reduce tissue necrosis. It needs 2-3 weeks until reconstructive procedures. This long process increases the financial burden on patients as well as the risk of infection. So, finding an effective therapeutic method is of great concern.

In recent years, stem cells such as bone marrowderived stem cells (BM-MSCs) (Zheng et al. 2008), ADSCs (Lu et al. 2008) and EPCs (Tepper et al. 2003) were all proved to be effective at increasing the formation of new blood vessels and improving the blood flow perfusion of ischemic flaps. However, bone marrow is the main source of BM-MSCs and EPCs, and the harvest of these cells may be associated with considerable morbidity at the donor site. Moreover, the limited reserve of BM-MSCs and EPCs necessitates in vitro expansion. Isolation of ADSCs is less morbidity, but in vitro expansion is still needed. Cell culture with 2-3 weeks is not only time-consuming, but also increases the risk of contamination and differentiation of stem cells.

Avoidance of in vitro culture makes BM-MNCs and SVF good alternatives for ischemia therapy. We have demonstrated that engraftment of BM-MNCs was effective at promoting neovascularization of flaps (Yang et al. 2010). So, based on our previous research experience of BM-MNCs, an effort was made to examine the effectiveness of SVF therapy on random skin flaps with BM-MNCs as positive control, and then to find a better alternate for clinical application.

To be byproduct of plastic surgery, adipose tissue is easily harvested with less invasion and exists as a better 

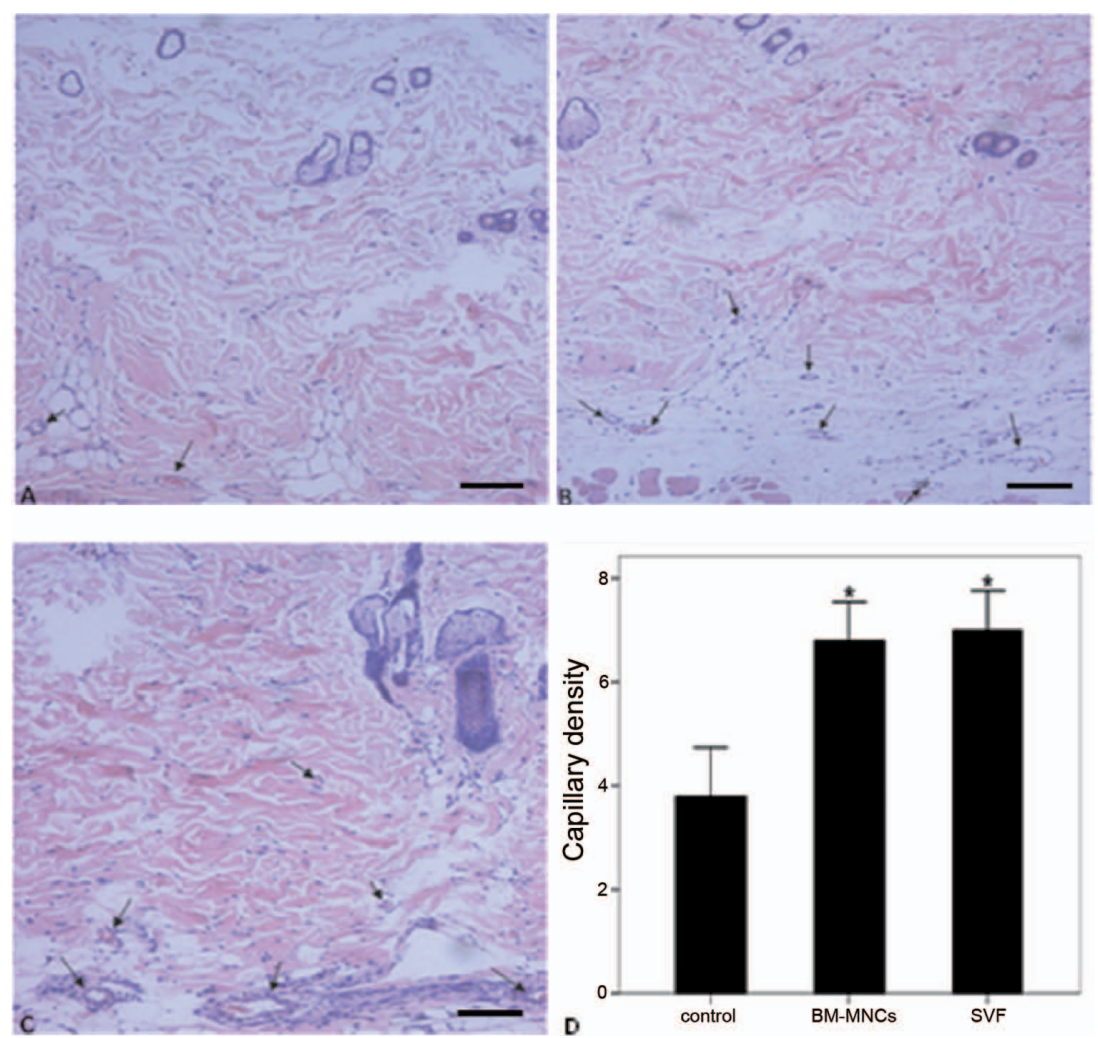

Fig. 3. H\&E staining of the flap tissue.

Tissue in the surviving district near the demarcation of survival and necrosis was harvested and fixed for H\&E staining to detect subcutaneous capillaries in the three groups. The results showed that the capillary density in BM-MNCs group (B) and SVF group (C) was significantly higher than that the control group (A), ${ }^{*} p<0.05$ (Original magnification: $100 \times)$. Each arrow indicates the capillaries in the subcutaneous tissue. Scale bar $=100 \mu \mathrm{m}$.
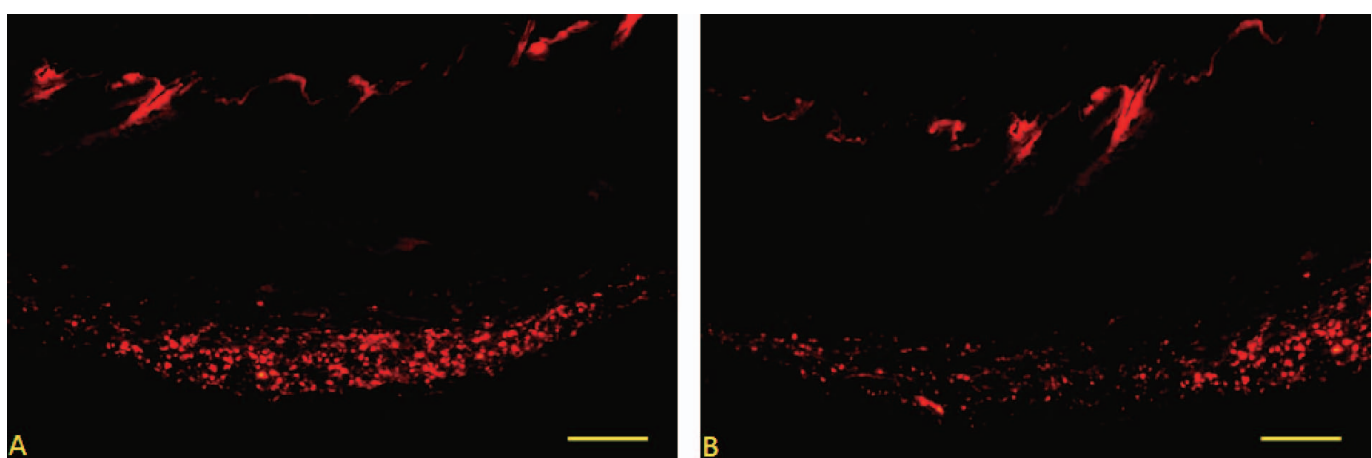

Fig. 4. DiI-positive cells in the flap tissues.

DiI-labeling cells were injected into flap tissues to observe the viability of engrafted cells in tissues on day 7 after operation. Under a fluorecent microscope, DiI-positive cells in flap tissue of SVF and BM-MNCs groups released specific red fluorecence and mainly stayed in subcutaneous tissue, signifying the engrafted cells survived in the tissue. A: DiIpositive cells in SVF group; B: DiI-positive cells in BM-MNCs group (Original magnification: $100 \times$ ). Scale bar $=100$ $\mu \mathrm{m}$.

reserve of stem cells. Existence of vast amounts of fat make SVF harvested largely with easy operation. Within $\mathrm{SVF}, \mathrm{CD} 34^{+} / \mathrm{CD} 90^{+}$cells residing in a periendothelial location are mainly ADSCs. These cells could differentiate into endothelial cells and, when palced in methylcellulose, were capable of forming capillary-like structures producing a high level of VEGF (De Francesco et al. 2009). Sumi et al.
(Sumi et al. 2007) transplanted SVF of C57BL/6 mice into ischemic hindlimb, and discovered that SVF significantly augmented collateral development as determined by the restoration of blood perfusion and capillary density of the ischemic muscle. Based on the positive findings in previous studies, SVF may be a better choice for the clinical treatment of ischemia therapy. 

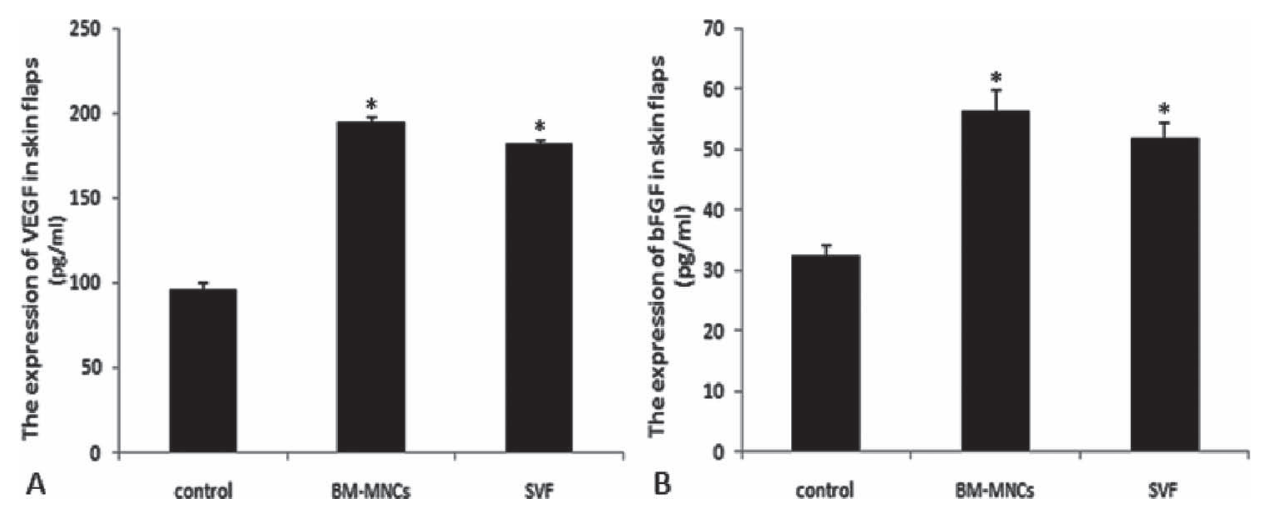

Fig. 5. ELISA assay of VEGF and bFGF in the flap tissues 7 days after surgery.

A: The expression of VEGF in the flaps of BM-MNCs group and SVF group was up-regulated and was higher than that in the control group. B: The mean bFGF level in the control group was statistically lower than the other two groups ( $n=6 ; * p<0.05$ when SVF group and BM-MNCs group compared with the control group).

In our study, using a standard rat random skin flap model, we demonstrated for the first time that local injection of SVF had similar effect on improving skin flap survival as BM-MNCs transplantation supported by improvement of neovascularization by virtue of increasing capillary angiogenesis and augmenting blood flow perfusion. These results were accordant with Sumi's study. He found angiogenic effects of SVF were as potent as those of BM-MNCs on ischemic legs (Sumi et al. 2007).

To observe the viability of engrafted cells, DiI was used to label cells. Seven days postoperative, the injected cells released specific red fluorescence. This result manifested that there was no immune rejection to the injected cells and the injected cells survived in flap tissues. The engrafted cells differentiating into vascular cells are considered to be one of mechanisms of SVF therapy (Sumi et al. 2007). However, as we know, the survival percentage of the engrafted cells was very low (Wu et al. 2007), not to mention playing main roles in neovascularization. So, many researchers believed that the indirect effects of angiogenic growth factors released from the engrafted cells contributed largely to the improved neovascularization.

In present study, we examined protein expression levels of VEGF and bFGF in the flaps. VEGF and bFGF are now considered to be the most important factors in angiogenesis (Ferrara and Alitalo 1999). bFGF can activate EPCs and then initiates neovascularization (Hebda et al. 1990). VEGF plays an important role in regulating the proliferation of endothelial cells and inhibiting apoptosis exerted by VEGF activating anti-apoptotic kinase and maintaining survival signals (Grosjean et al. 2006; Liu et al. 2002). VEGF also could recruit stem cells from surrounding tissue and bone marrow to the ischemic tissue ( $\mathrm{Wu}$ et al. 2007). In our study, higher protein levels of VEGF and bFGF detected by ELISA showed in both SVF and BM-MNCs groups. Therefore, secretion of proangiogenic factors may be an important mechanism of the treatment.

We transplanted SVF and BM-MNCs into flap tissues 2 days before elevating flaps, which is different from Lu's study (Lu et al. 2008). In Lu's study, ADSCs were transplanted at the moment of flap elevation, and flap survival increased obviously. However, Simman et al. (Simman et al. 2005) found the effect of mesenchymal stem cells injected 1 week before operation was significantly better on ischemic flaps than the cells engrafted immediately at the moment of elevation. Flap elevation creats a hypoxic environment, and may decrease the survival ratio of engrafted cells. Cell injection before surgery provides the cells enough nutrition to survive and adequate time to proliferate and differentiate to balance the damage caused by hypoxia. So, combined with our previous experience, we transplanted cells into flap tissue 2 days before operation.

\section{Conclusions}

This study demonstrates that increased skin flap survival could be achieved by local application of SVF by secreting proangiogenesis factors such as VEGF and bFGF. With abundant reserve in adipose tissue and without morbidity at the donor site as well as the avoidance of in vitro culture, SVF therapy may be a promising therapy and deserves further investigation.

\section{Acknowledgments}

This work was supported by the National Natural Science Foundation of China (No. 30730092, 30925034), National Science Fund for Distinguished Young Scholars (No. 30925034).

\section{Conflict of Interest}

The authors report no conflict of interest.

\section{References}

Bunnell, B.A., Flaat, M., Gagliardi, C., Patel, B. \& Ripoll, C. (2008) Adipose-derived stem cells: isolation, expansion and differentiation. Methods, 45, 115-120.

De Francesco, F., Tirino, V., Desiderio, V., Ferraro, G. \& D'Andrea, F. (2009) Human CD34+/CD90+ ASCs are capable of growing as sphere clusters producing high levels of VEGF and forming capillaries. PLoS ONE, 4, e6537-6550.

Ferrara, N. \& Alitalo, K. (1999) Clinical applications of angio- 
genic growth factors and their inhibitors. Nat. Med., 5, 13591364.

Grosjean, J., Kiriakidis, S., Reilly, K., Feldmann, M. \& Paleolog, E. (2006) Vascular endothelial growth factor signalling in endothelial cell survival: a role for NFkappaB. Biochem. Biophys. Res. Commun., 340, 984-994.

Haws, M.J., Erdman, D., Bayati, S., Brown, R.E. \& Russell, R.C. (2001) Basic fibroblast growth factor induced angiogenesis and prefabricated flap survival. J. Reconstr. Microsurg., 17, 39-42.

Hebda, P.A., Klingbeil, C.K., Abraham, J.A. \& Fiddes, J.C. (1990) Basic fibroblast growth factor stimulation of epidermal wound healing in pigs. J. Invest. Dermatol., 95, 626-631.

Henry, T.D., Annex, B.H., McKendall, G.R., Azrin, M.A., Lopez, J.J., Giordano, F.J., Shah, P.K., Willerson, J.T., Benza, R.L., Berman, D.S., Gibson, C.M., Bajamonde, A., Rundle, A.C., Fine, J. \& McCluskey, E.R. (2003) The viva trial: vascular endothelial growth factor in ischemia for vascular angiogenesis. Circulation, 107, 1359-1365.

Liu, W.L., Guo, X., Chen, Q.Q. \& Guo, Z.G. (2002) VEGF protects bovine aortic endothelial cells from TNF-alpha- and H2O2-induced apoptosis via co-modulatory effects on p38-and p42/p44-CCDPK signaling. Acta Pharmacol. Sin., 23, 45-49.

Lu, F., Mizuno, H., Uysal, C.A., Cai, X., Ogawa, R. \& Hyakusoku, H. (2008) Improved viability of random pattern skin flaps through the use of adipose-derived stem cells. Plast. Reconstr. Surg., 121, 50-58.

Nall, A.V., Brownlee, R.E., Colvin, C.P., Schultz, G., Fein, D., Cassisi, N.J., Nguyen, T. \& Kalra, A. (1996) Transforming growth factor beta 1 improves wound healing and random flap survival in normal and irradiated rats. Arch. Otolaryngol. Head Neck Surg., 122, 171-177.

Rehman, J., Traktuev, D., Li, J., Merfeld-Clauss, S., Temm-Grove, C.J., Bovenkerk, J.E., Pell, C.L., Johnstone, B.H., Considine,
R.V. \& March, K.L. (2004) Secretion of angiogenic and antiapoptotic factors by human adipose stromal cells. Circulation, 109, 1292-1298.

Simman, R., Craft, C. \& Mckinney, B. (2005) Improved survival of ischemic random skin flaps through the use of bone marrow nonhematopoietic stem cells and angiogenic growth factors. Ann. Plast. Surg., 54, 546-552.

Sumi, M., Sata, M., Toya, N., Yanaga, K., Ohki, T. \& Nagai, R. (2007) Transplantation of adipose stromal cells, but not mature adipocytes, augments ischemia-induced angiogenesis. Life Sci., 80, 559-565.

Tepper, O.M., Galiano, R.D., Kalka, C. \& Gurtner, G.C. (2003) Endothelial progenitor cells: the promise of vascular stem cells for plastic surgery. Plast. Reconstr. Surg., 111, 846-854.

Wu, Y., Chen, L., Scott, P.G. \& Tredget, E.E. (2007) Mesenchymal stem cells enhance wound healing through differentiation and angiogenesis. Stem. Cells, 25, 2648-2659.

Yang, M., Sheng, L., Li, H., Weng, R. \& Li, Q.F. (2010) Improvement of the skin flap survival with the bone marrow-derived mononuclear cells transplantation in a rat model. Microsurgery, 30, 275-281.

Zhang, F., Oswald, T., Lin, S., Cai, Z., Lei, M., Jones, M., Angel, M.F. \& Lineaweaver, W.C. (2003) Vascular endothelial growth factor (VEGF) expression and the effect of exogenous VEGF on survival of a random flap in the rat. Br. J. Plast. Surg., 56, 653-659.

Zheng, Y., Yi, C., Xia, W., Ding, T., Zhou, Z., Han, Y. \& Guo, S. (2008) Mesenchymal stem cells transduced by vascular endothelial growth factor gene for ischemic random skin flaps. Plast. Reconstr. Surg., 121, 59-69.

Zimmerlin, L., Donnenberg, V.S., Pfeifer, M.E., Meyer, E.M., Peault, B., Rubin, J.P. \& Donnenberg, A.D. (2010) Stromal vascular progenitors in adult human adipose tissue. Cytometry $A, 77,22-30$. 